\title{
Manejo de malezas en las papas ${ }^{1}$
}

\section{Peter Dittmar, Seth Byrd, Lincoln Zotarelli, Diane Rowland, and William Stall ${ }^{2}$}

Las malezas causan problemas en la producción de patata en Florida y puede reducir rendimientos a través de competencia directa por la luz, la humedad y los nutrientes, o por albergar insectos y enfermedades que atacan las patatas. Las malezas pueden tener un impacto perjudicial sobre el rendimiento de los tubérculos comparado con aquellos tubérculos en condiciones sin maleza (Love et al. 1995; Nelson y Thoreson 1981). Por ejemplo, Love et al. (1995) consiguió una reducción en la biomasa vegetativa, la biomasa del tubérculo y el rendimiento total en las variedades 'Frontera Russet' y 'Russet Burbank' comparado con parcelas libres de maleza. Patatas con malezas también resultaron en un numero mayor de tubérculos pequeños y en menos tubérculos de grado No. 1 de USA en comparación con papas sin malezas (Love et al. 1995). Nelson y Thoreson (1981) encontraron que la competencia con las malezas reduce el tamaño del tubérculo y el número de tubérculos. Sin embargo, en ambos estudios, hubo poco efecto en la competencia con la maleza en la gravedad específica (Love et al. 1995; Nelson y Thoreson 1981). Además de los impactos en la formación fisiológica de la papa propia, malezas presente durante la cosecha puede aumentar daños mecánicos a los tubérculos y reducir la eficiencia de la cosecha al retardar la operación, dejando los tubérculos por encima del suelo.

La competencia con las malezas poco después del sembrado es extremadamente crítica; por lo tanto hay que enfatizar las medidas de control de malezas durante este período.
Las patatas pueden ser cultivadas durante un período de 7-meses en Florida, y durante este período las condiciones climáticas variables influyen en la diversidad y la gravedad de las especies de maleza presente. Los productores deben planear un programa de control total de la maleza que integre los métodos químicos, mecánicos y culturales que se ajusten a las especies de maleza presentes y a las prácticas de producción.

Un método cultural que puede tener un impacto en el control de la maleza es el espaciado entre hileras. Love et al. (1995) comparó el efecto de dos espacios de siembra (28" y 37.8 ") en relación a la competencia con la malezas y no encontró diferencia alguna en el rendimiento de la biomasa de la maleza o del rendimiento del tubérculo. Esto pudo haber estado relacionado con la conclusión de que la distancia entre hileras no afecta mucho a la hora del cierre del dosel, lo que impacta el establecimiento de la maleza. También compararon el efecto de espacio entre las semillas dentro de la hilera (5.9", 9.8”, y 13.8") y se encontró una pequeña disminución numérica en la biomasa de las malezas con la disminución de espacio entre semillas, probablemente a causa del alargamiento vegetativo más rápido y un mayor impacto en el cierre del follaje (Love et al. 1995).

Más allá de la hilera y el espaciado entre planta, los investigadores han estudiado otras prácticas para el control de la maleza, tales como cultivos de cobertura. Boydston y Hang (1995) han estudiado el efecto del cultivo de colza en la

1. Este documento, HS1213, es uno de una serie de publicaciones del Horticultural Sciences, Servicio de Extensión Cooperativa de la Florida, Instituto de Alimentos y Ciencias Agrícolas, Universidad de la Florida. (UF/IFAS). Fecha de primera publicación: January 2013. Visite nuestro sitio web EDIS en $<$ http://edis.ifas.ufl.edu>. This publication is a translation of HS194, Weed Management in Potato.

2. Peter Dittmar, assistant professor, Horitcultural Sciences Department; Seth Byrd, graduate student, Agronomy Department; Lincoln Zotarelli, assistant professor, Horticultural Sciences Department; Diane Rowland, associate professor, Agronomy Department: and William Stall, emeritus professor, Horticultural Sciences Department, Cooperative Extension Service, Institute of Food and Agricultural Sciences, University of Florida, Gainesville, FL 32611.

The Institute of Food and Agricultural Sciences (IFAS) is an Equal Opportunity Institution authorized to provide research, educational information and other services only to individuals and institutions that function with non-discrimination with respect to race, creed, color, religion, age, disability, sex, sexual orientation, marital status, national origin, political opinions or affiliations. U.S. Department of Agriculture, Cooperative Extension Service, University of Florida, IFAS, Florida A\&M University Cooperative

Extension Program, and Boards of County Commissioners Cooperating. Nick T. Place, Dean 
presencia de malezas cuando ésta se siembra anteriormente a la papa con la presencia de la maleza. La colza contiene un compuesto llamado glucosinolato que causan efectos negativos en la germinación de la semilla de malezas (Evenari 1949).

En un estudio de 2 años comparando la colza y el pasto Sudán como cultivos de cobertura, la colza produce más biomasa y obtuvo mayor reducción en la densidad de maleza en la siguiente cosecha de patata en comparación con pasto Sudán (Boydston y Hang, 1995). Las patatas sembradas después de la colza (con o sin herbicida) tuvieron mayor rendimiento que las papas sembradas después del pasto Sudán mientras que la gravedad específica de los tubérculos no fue afectada por ninguno de los dos cultivos de cobertura (Boydston y Hang 1995).

La cultivacion es otra manera efectiva de manejar la maleza desde temprano en la temporada de cultivo, cuando la presencia de la maleza es más perjudicial para el crecimiento y el rendimiento de la patata (Connell, Binning, y Schmitt 1999; Nelson y Thoreson 1981). Se ha demostrado que las malezas que emergen (plural, there are more than just one weed) antes que la papa son las más competitivas con el cultivo (Love et al. 1995). Connell, Binning, y Schmitt (1999) han determinado que la dossel de la patata seguirá dándole sombra a las malezas que emerjan después del cultivo inicial y de las aplicaciones de los herbicidas, 7-8 semanas después de la aparición de la cosecha. Esta línea de tiempo puede variar según la variedad de papa debido características propias en la estructura del dosel. Además si la cobertura vegetal está dañada, aplicaciones de herbicida adicional pueden ser necesarias hasta que se recupere (Connell, Binning, y Schmitt 1999). Durante las operaciones de aporque, cultivadores rodantes detrás de las cuchillas de aporque pueden desarraigar mucha maleza anual que pudo haber sobrevivido a los herbicidas de pre-siembra. El cultivo y el aporque, mientras útil, también pueden afectar la eficiencia de varios herbicidas aplicados por suelo. Para los variedades de papa que requieren varias operaciones de aporque durante la temporada, herbicidas adicionales pueden ser necesarias durante o directamente después del aporque. Esta combinación de practicas puede mejorar y extender el control de la maleza durante la temporada de cultivo.
La efectividad del herbicida depende del clima, riego, propiedades del suelo, la selección apropiada para las especies de maleza a ser controladas, la aplicación precisa de los herbicidas y el momento oportuno de aplicación. Para obtener resultados consistentes, hay que leer la etiqueta del herbicida y leer otras fuentes de información acerca de la aplicación y el tiempo apropiado de cada unos de los herbicidas. Para evitar la confusión entre fórmulas comerciales, a continuación hay una lista de las tasas sugeridas en la Tabla 1 que se exponen en libras de ingrediente activo por acre (lb. a.i./A). Para los suelos franco y los arenosos con baja materia orgánica, las tasas más bajas deben ser usadas. Todos los herbicidas a continuación han sido probados en ensayos de investigación en Florida con resultados exitosos.

Al aplicar un herbicida por primera vez en una nueva área, éste deber ser probado primero en un área pequeña. Antes de aplicar un herbicida, lea cuidadosamente y siga la etiqueta.

\section{Referencias}

Boydston, R. A., and A. Hang. 1995. "Rapeseed (Brassica napus) Green Manure Crop Suppresses Weeds in Potato (Solanum tuberosum)." Weed Technology 9 (4): 669-675.

Connell, T. R., L. K. Binning, and W. G. Schmitt. 1999. "A Canopy Development Model for Potatoes." American Journal of Potato Research 76 (3): 153-159.

Evenari, M. 1949. “Germination Inhibitors.” The Botanical Review 15 (3): 153-194.

Love, S. L., C. V. Eberlein, J. C. Stark, and W. H. Bohl. 1995. "Cultivar and Seedpiece Spacing Effects on Potato Competitiveness with Weeds." American Journal of Potato Research 72 (4): 197-213.

Nelson, D. C., and M. C. Thoreson. 1981. "Competition Between Potatoes (Solanum tuberosum) and Weeds." Weed Science 29 (6): 672-677. 
Tabla 1. Control químico de maleza pre-emergente en la papa

\begin{tabular}{|c|c|c|}
\hline $\begin{array}{l}\text { Nombre Común lb. a.i. } \\
\text { /A }\end{array}$ & $\begin{array}{l}\text { (Nombre Comercial) } \\
\text { monto del producto/A }\end{array}$ & Maleza a controlar / Observaciones \\
\hline $\begin{array}{l}\text { Carfentrazone } \\
\text { Hasta } 0.031\end{array}$ & $\begin{array}{l}\left(\text { Aim }^{\circledast}\right) 2 \text { EC o } 1.9 \\
\text { EW Hasta } 2 \text { fl. oz. }\end{array}$ & $\begin{array}{l}\text { Para maleza de hoja ancha saliente. Aplique como quemante para la maleza de } \\
\text { hoja ancha emergida. Use el aceite concentrado para cultivo (COC) o surfactante } \\
\text { no iónico (NIS) en cantidades recomendadas. Puede ser mezclado en un tanque } \\
\text { con otros herbicidas. }\end{array}$ \\
\hline $\begin{array}{l}\text { Dimetenamida-p } \\
0.56-0.84\end{array}$ & $\begin{array}{l}\left(\text { Outlook }^{\oplus}\right) 6 \mathrm{~L} \\
12-8 \text { fl. oz. }\end{array}$ & $\begin{array}{l}\text { Aplicar después de la siembra o de arrastrar. Consulte la etiqueta para las tasas } \\
\text { exactas basadas en la textura del suelo. En frío, o condiciones húmedas, el retraso } \\
\text { del crecimiento del cultivo puede ocurrir. PHI } 40 \text { días. }\end{array}$ \\
\hline $\begin{array}{l}\text { EPTC } \\
1\end{array}$ & $\begin{array}{l}\left(\text { Eptam }^{\oplus}\right) 20 \mathrm{G} \\
15 \mathrm{lb} .\end{array}$ & $\begin{array}{l}\text { Para maleza de hoja ancha y gramíneas y coquillo. Aplicar en la pre-planta } \\
\text { o después del pase de rastra. Incorporar mecánicamente o con riego a una } \\
\text { profundidad de } 2-4 \text { pulgadas, No aplique la formula emulsionable pre- } \\
\text { emergente para patatas en el invierno o en el inicio de la primavera. }\end{array}$ \\
\hline $\begin{array}{l}\text { Flumioxazina } \\
0.05\end{array}$ & $\begin{array}{l}\left(\text { Chateau }^{\oplus}\right) 51 \text { WDG } \\
1.5 \text { oz. }\end{array}$ & $\begin{array}{l}\text { Para maleza de hoja ancha anual. Aplicar después del aporque con un mínimo de } \\
2 \text { pulg. de suelo cubriendo la porción vegetativa de la planta. Debe ser mezclada } \\
\text { con otros herbicidas, especialmente para el control de gramíneas. }\end{array}$ \\
\hline $\begin{array}{l}\text { Fomesafen } \\
0.25\end{array}$ & $\begin{array}{l}\left(\text { Reflex }^{\oplus}\right) 2 \mathrm{EC} \\
1.0 \text { pt. }\end{array}$ & $\begin{array}{l}\text { Para maleza de hoja ancha y gramíneas. La efectividad se reduce si las prácticas } \\
\text { culturales exponen el suelo sin tratar. Puede ser aplicado después del pase de } \\
\text { rastra o antes de que los cultivos broten. La tolerancia de variedad puede variar; } \\
\text { ensaye en una muestra pequeña antes de aplicar a todo el terreno. PHI } 70 \text { días. }\end{array}$ \\
\hline Glyphosate & $\begin{array}{l}\text { (Varias formulas) } \\
\text { Consulte las etiquetas }\end{array}$ & $\begin{array}{l}\text { Para maleza de hoja ancha crecida o gramíneas. Aplicar como quemante previo } \\
\text { a la emergencia del cultivo. Consulte la etiqueta para las direcciones individuales } \\
\text { del producto. }\end{array}$ \\
\hline $\begin{array}{l}\text { Linuron } \\
0.75-1.25\end{array}$ & $\begin{array}{l}\left(\text { Lorox }^{\circledR} \mathrm{DF}\right) 50 \mathrm{DF} \\
1.5-2.5 \mathrm{lb} .\end{array}$ & $\begin{array}{l}\text { Aplique después del pase de rastra. Si la maleza está presente, incluya un NIS. Los } \\
\text { mejores resultados son obtenidos en campos húmedos en aplicaciones seguida } \\
\text { por lluvia o por riego en un rango no mayor a } 2 \text { semanas. }\end{array}$ \\
\hline $\begin{array}{l}\text { S-metolachlor } \\
0.95-1.9\end{array}$ & $\begin{array}{l}\left(\text { Dual Magnum }{ }^{\circledast}\right) \\
7.62 \mathrm{EC} \\
1.0-2.0 \text { pt. }\end{array}$ & $\begin{array}{l}\text { Para maleza de hoja ancha anual y gramíneas. Aplicar después del pase de rastra } \\
\text { y maleza crecida. Si es frío, el suelo mostrará condiciones húmedas después de la } \\
\text { aplicación, puede que retrase la madurez o reduzca el rendimiento de la cosecha. } \\
\text { PHI } 60 \text { días. }\end{array}$ \\
\hline $\begin{array}{l}\text { Metribuzin } \\
0.23-1.0\end{array}$ & $\begin{array}{l}\left(\text { Tricor }^{\oplus} \mathrm{DF}\right) 75 \mathrm{DF} \\
0.3-1.3 \mathrm{lb} .\end{array}$ & $\begin{array}{l}\text { Para maleza de hojas anchas anual. Aplicar después del pase de rastra pero antes } \\
\text { de que los cultivos emerjan. No incorpore. Use tasas bajas en suelos arenosos. }\end{array}$ \\
\hline $\begin{array}{l}\text { Metribuzin }+ \\
\text { S-metolachlor } \\
0.23-0.31+ \\
0.98-1.31\end{array}$ & $\begin{array}{l}\left(\text { Boundary }{ }^{\circledast}\right) 6.5 \text { EC } \\
1.5-2 \text { pt. }\end{array}$ & $\begin{array}{l}\text { Para maleza de hoja ancha y gramíneas. Aplicar antes de que la patata crezca, } \\
\text { incluyendo el pase de rastra. No incorporar. }\end{array}$ \\
\hline $\begin{array}{l}\text { Paraquat } \\
0.25-0.5\end{array}$ & $\begin{array}{l}\left.\text { (Gramoxone }{ }^{\oplus} \mathrm{SL}\right) 2 \mathrm{SL} \\
1.0-2.0 \mathrm{pt.} \\
\left.\text { (Firestorm }{ }^{\oplus}\right) 3 \mathrm{SL} \\
0.7-1.3 \text { pt. }\end{array}$ & $\begin{array}{l}\text { Para maleza de hoja ancha crecida y gramíneas. Aplicar como pre-planta para el } \\
\text { tratamiento de incendio. Aplicar hasta en el agrietamiento del suelo antes que las } \\
\text { patatas crezcan. Use NIS. }\end{array}$ \\
\hline Ácido Pelargonic & $\begin{array}{l}\left(\text { Scythe }{ }^{\circledast}\right) 4.2 \text { EC } \\
3 \%-10 \% \text { v/v }\end{array}$ & $\begin{array}{l}\text { Para maleza de hoja ancha y gramíneas. Es un producto de contacto, no selectivo, } \\
\text { aplicado foliarmente y sin control residual. Puede ser mezclado en un tanque con } \\
\text { compuestos residuales del suelo. }\end{array}$ \\
\hline $\begin{array}{l}\text { Pendimethalin } \\
0.75\end{array}$ & $\begin{array}{l}\left(\text { Prowl }^{\circledR} \mathrm{H}_{2} \mathrm{O}\right) 3.8 \\
1.5 \text { pt. }\end{array}$ & $\begin{array}{l}\text { Para maleza de hoja ancha y gramíneas. Aplicar después de cultivar pero antes de } \\
\text { que las papas y la maleza crezcan o después del pase de rastra. Incorporar con la } \\
\text { lluvia o mecánicamente en la parte superior 1-2 pulgadas. }\end{array}$ \\
\hline $\begin{array}{l}\text { Pyraflufen } \\
0.0008-0.003\end{array}$ & $\begin{array}{l}\left(\mathrm{ET}^{\oplus} \text { Herbicida/Defoliant) }\right. \\
0.208 \mathrm{EC} \\
0.5-2.0 \text { fl. oz. }\end{array}$ & $\begin{array}{l}\text { Para maleza de hoja ancha crecida. Aplicar como un tratamiento de quema antes } \\
\text { de la siembra. }\end{array}$ \\
\hline $\begin{array}{l}\text { Rimsulfuron } \\
0.016-0.023\end{array}$ & $\begin{array}{l}\left(\text { Matrix }^{\circledast}\right) 25 \text { DF } \\
1-1.5 \text { oz. }\end{array}$ & $\begin{array}{l}\text { Aplicar inmediatamente después del arrastre. Lluvia o riego por aspersión de 1/3 } \\
-1 \text { pulgada. Es requerido en } 5 \text { días después de la aplicación. No exceda } 2.0 \text { oz./A } \\
\text { por año }\end{array}$ \\
\hline
\end{tabular}


Tabla 2. Control químico de maleza post emergente en la papa

\begin{tabular}{|c|c|c|}
\hline Ingrediente Activo lb. a.i. /A & $\begin{array}{l}\text { (Nombre Comercial) } \\
\text { cantidad del producto/A }\end{array}$ & Maleza por controlar / Observaciones \\
\hline $\begin{array}{l}\text { Carfentrazone } \\
\text { Hasta } 0.31\end{array}$ & $\begin{array}{l}\left(\text { Aim }^{\circledast}\right) 2 \text { EC o } 1.9 \text { EW } \\
\text { Hasta } 2 \text { oz. }\end{array}$ & $\begin{array}{l}\text { Para maleza de hoja ancha. Use un aceite concentrado para cultivos } \\
\text { (COC) o un surfactante no iónico (NIS) a tasas recomendadas. Puede } \\
\text { ser mezclado en un tanque con otros herbicidas. No exceda de } 6.1 \text { fl. } \\
\text { oz. por temporada de cultivo. PHI } 7 \text { días. }\end{array}$ \\
\hline $\begin{array}{l}\text { Clethodim } \\
0.09-0.25 \\
0.07-0.25\end{array}$ & $\begin{array}{l}\left(\text { Select }^{\oplus}, \text { Arrow }^{\circledast}\right) 2 \text { EC } \\
6-16 \text { fl. oz. } \\
(\text { Select Max } \\
\end{array}$ & $\begin{array}{l}\text { Perennes y gramíneas anuales. En campos con alta presión de pasto } \\
\text { o gramíneas más altas, utilice tasa más altas o repita las aplicación } \\
\text { cada } 14 \text { días. Utilice un COC a } 1 \% \text { v/v en acabado de aerosol en } \\
\text { volumen. Un surfactante no-iónico con Select Max }{ }^{\circledast} \text {. PHI } 30 \text { días. }\end{array}$ \\
\hline EPTC & $\begin{array}{l}\left(\text { Eptam }^{\oplus}\right) 20 \mathrm{G} \\
15 \mathrm{lb} .\end{array}$ & $\begin{array}{l}\text { Para maleza de hoja ancha y gramíneas y coquillo. Aplicar en layby a } \\
\text { un suelo limpio y cultivado Incorporar mecánicamente o por riego. } \\
\text { PHI } 45 \text { días. }\end{array}$ \\
\hline $\begin{array}{l}\text { Metribuzin } \\
0.23-0.5\end{array}$ & $\begin{array}{l}\left(\text { Tricor }^{\oplus}\right) 75 \mathrm{DF} \\
0.3-0.6 \mathrm{lb} .\end{array}$ & $\begin{array}{l}\text { Para maleza de hojas anchas. No aplicar a principios de piel suave, } \\
\text { piel roja y otras variedades indicadas en la etiqueta. No exceda } 1.3 \text { lb. } \\
\text { por producción/A. PHI } 60 \text { días. }\end{array}$ \\
\hline Ácido Pelargonic & $\begin{array}{l}\left(\text { Scythe }{ }^{\circledast}\right) 4.2 \text { EC } \\
3 \%-10 \% \text { v/v }\end{array}$ & $\begin{array}{l}\text { Para maleza de hoja ancha crecida y gramíneas. Dirija el rocío a } \\
\text { las mitades entre hileras. El producto es en contacto, no-selectivo, } \\
\text { herbicida de aplicación foliar sin control residual. Puede ser } \\
\text { mezclado con varios compuestos residuales del suelo. }\end{array}$ \\
\hline $\begin{array}{l}\text { Rimsulfuron } \\
0.016-0.023\end{array}$ & $\begin{array}{l}\left(\text { Matrix }{ }^{\circledast}\right) 25 \mathrm{DF} \\
1-1.5 \mathrm{oz} .\end{array}$ & $\begin{array}{l}\text { Aplicar inmediatamente después del pase de rastra. Lluvia o riego } \\
\text { por aspersión de } 1 / 3-1 \text { pulgada. Es requerido dentro de } 5 \text { días } \\
\text { después de la aplicación. Aplicar como un tratamiento secuencial } \\
\text { 14-28 días después de la primera aplicación. No exceder } 2.0 \text { oz./A } \\
\text { por año. }\end{array}$ \\
\hline $\begin{array}{l}\text { Sethoxydim } \\
0.28-0.47\end{array}$ & $\begin{array}{l}\left(\text { Poast }^{\oplus}\right) 1.5 \mathrm{EC} \\
1.5-2.5 \mathrm{pt} .\end{array}$ & $\begin{array}{l}\text { Controla el crecimiento de las gramíneas. Un total de } 5.0 \text { pt./A } \\
\text { aplicadas en una temporada. Incluya una COC. Los resultados } \\
\text { insatisfactorios pueden ocurrir si es aplicada al pasto bajo estrés bajo } \\
\text { estrés. PHI } 30 \text { días. }\end{array}$ \\
\hline
\end{tabular}

